INPLASY

PROTOCOL

To cite: Zhou et al. Early prediction models for prognosis of diabetic ketoacidosis in the Emergency Department. Inplasy protocol 202130086. doi:

10.37766/inplasy2021.3.0086

Received: 22 March 2021

Published: 23 March 2021

Corresponding author: Yao Chen

815485358@qq.com

Author Affiliation:

Emergency Medical

Laboratory, West China

Hospital, Sichuan University

Support: Science Project of Sichuan.

Review Stage at time of this submission: Piloting of the study selection process.

Conflicts of interest:

None declared.

\section{Early prediction models for prognosis of diabetic ketoacidosis in the Emergency Department}

Zhou, Y1; Lv, L2; Chen, Y³.

Review question / Objective: P:Adult, children, pregnant women; I:Patients with diabetic ketoacidosis who have bad prognosis; C:Patients with diabetic ketoacidosis who have good prognosis; O:Risk of disease due to the diabetic ketoacidosis; S:Prospective/retrospective cohort study, RCT, etc.

Condition being studied: Diabetic ketoacidosis is one of the most serious and life-threatening hyperglycemia emergencies in diabetes. The patient with DKA can progress quickly within few hours, and even some patients have developed to critical state without being aware of it. Early evaluation of DKA prognosis is important. Up to now, there is no systematic review or meta-analysis of prognostic prediction models of diabetic ketoacidosis. We aim to systematically review and critically evaluate existing predictive models of diabetic ketoacidosis, especially the prognostic models of the disease.

INPLASY registration number: This protocol was registered with the International Platform of Registered Systematic Review and Meta-Analysis Protocols (INPLASY) on 23 March 2021 and was last updated on 23 March 2021 (registration number INPLASY202130086).

\section{INTRODUCTION}

Review question / Objective: P:Adult, children, pregnant women; I:Patients with diabetic ketoacidosis who have bad prognosis; C:Patients with diabetic ketoacidosis who have good prognosis;
O:Risk of disease due to the diabetic ketoacidosis; S:Prospective/retrospective cohort study, RCT, etc.

Condition being studied: Diabetic ketoacidosis is one of the most serious and life-threatening hyperglycemia 
emergencies in diabetes. The patient with DKA can progress quickly within few hours, and even some patients have developed to critical state without being aware of it. Early evaluation of DKA prognosis is important. Up to now, there is no systematic review or meta-analysis of prognostic prediction models of diabetic ketoacidosis. We aim to systematically review and critically evaluate existing predictive models of diabetic ketoacidosis, especially the prognostic models of the disease.

\section{METHODS}

Participant or population: Adult, children, pregnant women.

Intervention: Patients with diabetic ketoacidosis who have bad prognosis.

Comparator: Patients with diabetic ketoacidosis who have good prognosis.

Study designs to be included: Prospective/ retrospective cohort study, RCT, etc.

Eligibility criteria: We will collect all crosssectional studies that predict or analyzed the admission and death of patients with diabetic ketoacidosis. The study includes adolescent participants, but children or pregnant women doesn't meet the inclusion criteria. No restrictions will be placed on sex or gender, race, comorbidities, or other characteristics. Animal studies, cadaver studies, case reports, comments, letters, protocols, guidelines, unpublished articles, and review papers will be excluded. This search will be limited to reports in English, and for which full-text access is available. Participants who are included in the articles we selected should be diagnosed with diabetic ketoacidosis.

Information sources: At first, the collection of bibliographic data will be made in the electronic databases: PubMed, Web of science, EMBASE, Cochrane Library, Google scholar, CNKI, Wanfang and VIP. We use the available publications of the diabetic ketoacidosis living systematic review for a list of keywords. The words are considered: diabetic ketoacidosis, diagnostic, prognostic, prediction, prediction model, regression, score, artificial intelligence, algorithm, deep learning, machine learning. We make the search terms by combining the words above: \#1 diabetic ketoacidosis \#2 diagnostic OR imaging OR prognostic OR prognosis OR prediction OR prediction model OR mortality OR regression OR score OR artificial intelligence OR algorithm OR deep learning OR machine learning \#3 APACH II OR PSI ORSOFA OR qSOFA OR SAPS \#4 english NOT animal NOT meternal \#1 AND \#2 AND \#3 AND \#4.

Main outcome(s): Risk of disease due to the diabetic ketoacidosis.

Quality assessment / Risk of bias analysis: The Grading of Recommendations, Assessment, Development and Evaluation (GRADE) assessment tool will be used for conducting an appraisal of the studies' methodological quality. Every selected study will be evaluated by 2 reviewers independently, a third one as a consulter. The GRADE evaluation system included bias risk; heterogeneity; indirectness; imprecision; publication bias. And each level of evidence is divided into "very low", "low", "moderate", or "high" judgment.

Strategy of data synthesis: For qualified articles, we would like to combine the collected data according to characteristics of eligible trials. In line with the Cochrane guideline, we will express risk ratio with $95 \%$ confidence intervals $(95 \% \mathrm{Cl})$ using fixed effect model. Besides the random effect model will be used for continuous outcomes because of clinical heterogeneity. Statistical heterogeneity will be investigated using $x^{2}$ test and 12 statistic $(50 \%$, strong heterogeneity). We will assess possible publication bias using the Egger funnel plot. All data will be performed by using Review Manager (RevMan version 5.4.0) software and $P$ value $<.05$ will be considered statistically significant. 
Subgroup analysis: For qualified articles, we would like to combine the collected data according to characteristics of eligible trials. In line with the Cochrane guideline, we will express risk ratio with $95 \%$ confidence intervals $(95 \% \mathrm{Cl})$ using fixed effect model. Besides the random effect model will be used for continuous outcomes because of clinical heterogeneity. Statistical heterogeneity will be investigated using $x 2$ test and 12 statistic $(50 \%$, strong heterogeneity). We will assess possible publication bias using the Egger funnel plot. All data will be performed by using Review Manager (RevMan version 5.4.0) software and $P$ value $<.05$ will be considered statistically significant.

Sensitivity analysis: In line with the Cochrane guideline, we will express risk ratio with $95 \%$ confidence intervals $(95 \% \mathrm{Cl})$ using fixed effect model. Besides the random effect model will be used for continuous outcomes because of clinical heterogeneity. Statistical heterogeneity will be investigated using $x^{2}$ test and 12 statistic $(50 \%$, strong heterogeneity). We will assess possible publication bias using the Egger funnel plot. All data will be performed by using Review Manager (RevMan version 5.4.0) software and $P$ value $<.05$ will be considered statistically significant.

Country(ies) involved: China.

Keywords: Prediction models, prognosis, Diabetic ketoacidosis protocol, systematic review, meta-analysis, Emergency, early.

Contributions of each author:

Author 1 - Yiwu Zhou.

Email: 456zyw@163.com

Author 2 - Lin Lv.

Email: Ivlin202011@163.com

Author 3 - Yao Chen.

Email: 815485358@qq.com 